\title{
Study of the incidence of accidental dural punctures (ADP) related to the body mass index (BMI) in the Obstetric Anaesthesiology Service of the General University Hospital Gregorio Marañón
}

\section{Background}

Epidural technique for labor is an essential part in the pain management in the obstetric patient. In our center this procedure is performed in more than $90 \%$ deliveries attended. Despite being a safe technique, an accidental dural puncture (ADP) can take place. Published incidence ranges between 0.2 and $4 \%$ !

This complication can have important clinical consequences such as post- puncture headache (PPHA). ADP is related to a number of factors, amongst them, the needle's calibre, patient's age and weight etc. The goal of our study is to determine the relationship between the incidence of ADP and patient's BMI.

\section{Materials and Methods:}

We designed a descriptive, observational and crosssectional study where we collected information on $\mathbf{2 7 0 9 4}$ epidurals performed between June 2010 and March 2017. Before the epidural informed consent was obtained from all patients. All the procedures took place in the delivery rooms. Afterwards, a survey is filled in with the following data:
- Age
- ASA
- Weight and height
- Week of pregnancy and obstetric formula.

Later on, data related to the anesthetic technique is filled in: epidural or spinal technique, if the loss of ressistance was tested with air or saline solution, number of spaces punctured, puncture site, patient's position. Complications were also described: blood puncture, paresthesias, intravascular or painful injection, complete spinal block, or any others incidence.

Subsequently we calculated the patient's BMI which was then related with ADP incidence. BMI was classified as low BMI when under 18.5, normal between 18.5-24.99, overweight 25-29.99, obesity above 30 and morbid obesity above 40 .

\section{Results:}

Between June 2010 and May 2017, a total of 27094 women in labor received epidural analgesia. I47 ADPs were diagnosed, representing incidence of $\mathbf{0 . 5 4 \%}$. We collected weight and height data from 99 of these 147 patients with the following distribution:
Normal BMI we found 30 PDA (29.7\%), with a BMI of overweight there were 42 (4I.58\%), with obesity $25(\mathbf{2 4 . 7 5 \% )}$ and finally, 2 patients classified as morbid obesity (1.98\%).

\section{Discussion}

Epidural analgesia is still the technique of choice for woman in labor being safe and effective, although we must not forget that it is not complication free. The ADP can produce PPHA that conditions a great discomfort for the patient, in addition to the need for treatment that can range from intravenous or oral analgesia to the realization of a blood patch albeit increasing in-hospital stay.

Different risk factors have been identified ${ }^{2}$ in other studies which can condition an increase in the incidence of ADP, such as the the anesthesiologist's experience performing the technique, women's weight, as well as the ability to adopt the posture and remain still during the procedure, the needle's characteristics (Tuohy needle) ... Etc.

In our case, and regarding the patient's physical characteristics, we found that the majority of ADPs occur in those with a BMI above 25, with the highest incidence in the overweight group.

*As a protective measure against ADP, some authors suggest leaving the catheter in the intradural space to favour fibrosis and thus avoid cerebrospinal fluido leak, although there is no consensus in the literature regarding this technique's results.

\section{Incidence of ADP}

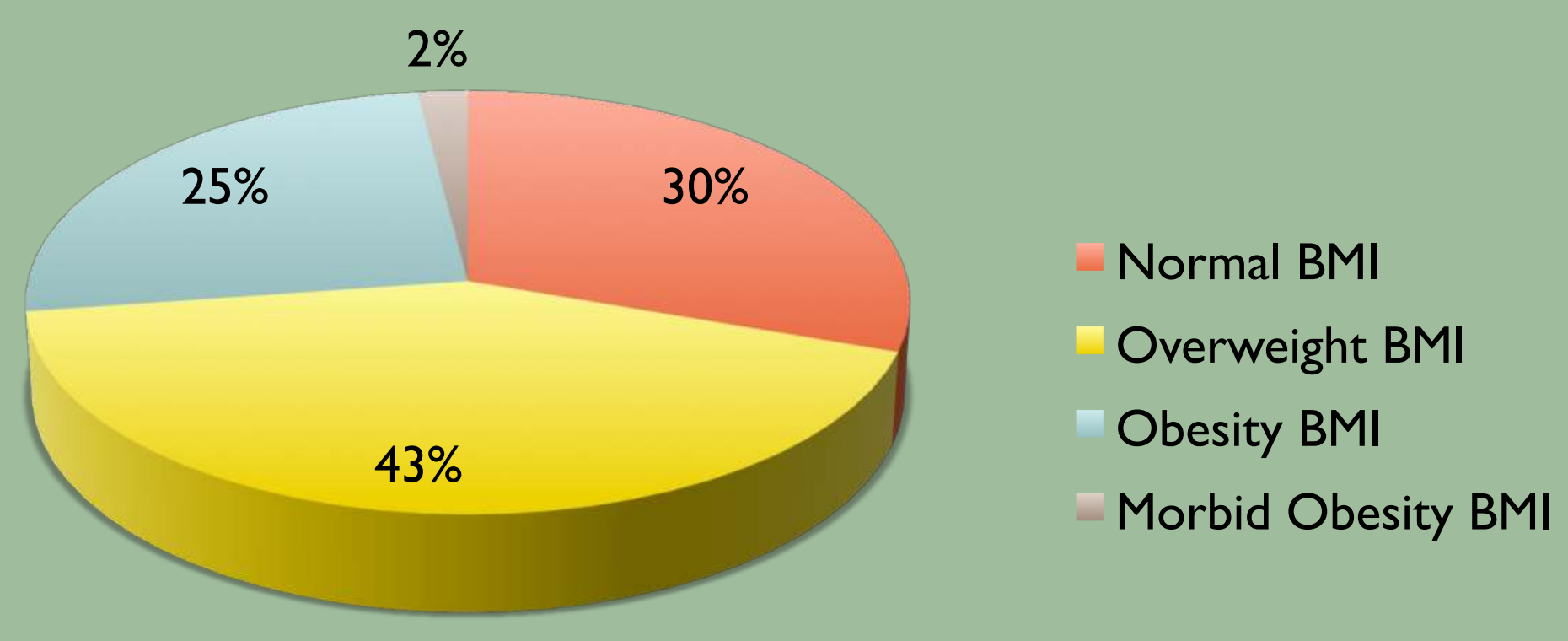

\section{Conclusions}

The incidence of ADP in our hospital is similar to previous series. With the data we have collected and analyzed, we can conclude that the rate of ADP is more frequent in patients with a BMI greater than 25 (68.3I\% of cases). 